\title{
EFEITO DA SUPLEMENTAÇÃO ORAL DE GLUTAMINA NA PERFORMANCE DE NADAADORES DE MEIO- FUNDO E FUNDO
}

\section{Idico luiz Pellegrinotti}

Universidade Metodista de Piracicaba, Piracicaba, São Paulo, Brasil

Marcelo de Castro Cesar

Universidade Metodista de Piracicaba, Piracicaba, São Paulo, Brasil

Marta Cecilia Soli Alves Rochelle

Universidade Metodista de Piracicaba, Piracicaba, São Paulo, Brasil

Sergio Luiz de Almeida Rochelle

Universidade Metodista de Piracicaba, Piracicaba, São Paulo, Brasil

João Paulo Borin

Universidade Estadual de Campinas, Campinas, São Paulo, Brasil

Reinaldo Rosa

Universidade Metodista de Piracicaba, Piracicaba, São Paulo, Brasil

Felipe Fredizzi Donatto

Universidade Metodista de Piracicaba, Piracicaba, São Paulo, Brasil

Jonato Prestes

Universidade Católica de Brasília, Brasília, Distrito Federal, Brasil

\section{João Bartholomeu Neto}

Faculdade de Educação Física de Gurupi, Gurupi, Tocantis, Brasil

\section{Cláudia Regina Cavaglieri}

Universidade Metodista de Piracicaba, Piracicaba, São Paulo, Brasil

\section{Resumo}

O objetivo deste estudo foi observar a influência da suplementação oral de glutamina sobre a performance física de nadadores de meio-fundo e fundo. Foram selecionados 10 atletas do sexo masculino, idade entre 13 e 18 anos, especialistas em provas de natação de 400 e/ou 800 metros. Os nadadores foram divididos em dois grupos: grupo placebo $(\mathrm{n}=5)$, com ingestão de $5 \mathrm{~g}$ /dia de amido de milho durante 30 dias; e grupo glutamina ( $\mathrm{n}=5)$, com ingestão de $5 \mathrm{~g} /$ dia de glutamina durante 30 dias. Os testes (nado crawl; 800 metros e limiar anaeróbio [Lan], utilizando-se a dosagem de lactato como componente do Lan) ocorreram antes e após a interven- 
ção. A análise dos resultados foi realizada por meio do teste $t$ para dados pareados e valor de $p \leq 0,05$. Não foram observadas diferenças significantes no desempenho dos atletas durante os testes de 800 metros e do Lan em ambos os grupos. Os resultados sugerem que a suplementação oral de glutamina não influenciou na performance de nadadores de meio-fundo e fundo.

Palavras-chave: Natação. Glutamina. Performance.

\section{Introdução}

$\mathrm{U}$ ma alimentação adequada é fundamental para qualquer indivíduo. No caso de atletas, a preocupação com a dieta é ainda maior, visto que a energia necessária para a prática esportiva é fornecida pelos nutrientes consumidos diariamente. Uma dieta balanceada, com nutrientes oferecidos de forma criteriosa e integrada a um programa adequado de treinamento é fundamental para um melhor desempenho esportivo (CURI, 2000).

Em se tratando de atletas adolescentes, os cuidados tornam-se ainda maiores, pois além do desgaste gerado pela competição, a adolescência é um período caracterizado por mudanças de diferentes magnitudes, ou seja, de natureza física, psicológica e fisiológica (DAMSGAARD et al., 2001; BAXTER-JONES; THOMPSON; MALINA, 2002; SILVA et al., 2004).

O gasto energético aumenta consideravelmente de acordo com o exercício praticado, a intensidade, a duração e as características individuais, tais sexo, idade, raça, compleição física, de maturação e da ingestão, absorção e aproveitamento dos macro e micronutrientes (LEHMKUHL et al., 1992; BAXTER-JONES; THOMPSON; MALINA, 2002; SILVA et al., 2004).

Os aminoácidos, constituidores das moléculas de proteína, desempenham um papel-chave na estrutura muscular e são um dos principais substratos energético no exercício. A glutamina é o aminoácido mais abundante no plasma, representando cerca de 20\% do total; nos tecidos, representa um total de 60\% (LEHMKUHL et al., 1992). A sua concentração plasmática é bastante elevada em relação aos demais aminoácidos ( 0,6 mM), no entanto, sofre redução em corridas de longa duração (CASTELL et al., 1997). Ainda, a glutamina presente na dieta não atinge a corrente sanguínea, pois é significativamente metabolizada pelas células absortivas no intestino (WALSH et al.,1998).

A utilização da glutamina pelo organismo de atletas de resistência e de força é no sentido de prover o anabolismo celular, reduzir o cata- 
bolismo e combater a imunossupressão (PERES, 2004). A suplementação oral desse aminoácido poderia aumentar a concentração sérica, bem como poupar os substratos energéticos musculares, fato que acarretaria na melhora do desempenho de atletas em exercícios de longa duração. Portanto, na dependência dos estoques de glicogênio estarem ótimos ou depletados antes do início do exercício, a proporção de necessidade de energia suprida pelas proteínas é estimada entre 5 e $20 \%$ (BAXTER-JONES; THOMPSON; MALINA, 2002).

Quando a demanda de glutamina no organismo aumenta, o tecido muscular eleva a produção e liberação desse aminoácido (WALSH et al., 1998). Sabe-se que a glutamina é fundamental para a funcionalidade do sistema imunológico, pois é o principal precursor de energia e de macromoléculas para o linfócito (CURY-BOAVENTURA et al., 2008; LAGRANHA, et al., 2008). Por essa razão, utiliza-se glutamina em dietas enterais e parenterais de pacientes hospitalizados e com doenças graves (PREISER et al., 2003). O uso da glutamina também é uma boa alternativa de tratamento para pacientes acometidos por sepse e queimaduras e em atletas com imunossupressão após exercício intenso (CASTELL, 2003).

Em atletas, Castell (2003) observou que a suplementação oral de cinco gramas de glutamina, após exercício intenso e de longa duração, em maratonistas, foi eficiente para manter a concentração sérica desse aminoácido ao final do exercício, aumentar a razão de linfócitos e ainda diminuir a incidência de infecções nos sete dias posteriores. O grupo placebo, nesse mesmo estudo de Castell (2003), apresentou 51\% de incidência de infecções, enquanto o grupo suplementado com glutamina apresentou apenas 19\%. Curi-Boaventura et al. (2008) ministraram glutamina ou placebo para corredores de média distância, maratonistas, ultramaratonistas e remadores, imediatamente após a competição ou sessão de treinamento intenso. Esses autores observaram que a suplementação diminuiu a incidência de infecções nos sete dias posteriores ao exercício, mas não alterou a concentração sérica desse aminoácido, bem como o número total de leucócitos em comparação aos atletas que receberam placebo. Outro estudo realizado com maratonistas não encontrou melhora no sistema imunológico após suplementação com glutamina (NIEMAN, 2007).

Os efeitos ergogênicos da suplementação de glutamina durante o exercício ainda não foram totalmente elucidados (FLAK et al., 2003; FONTANA; VALDES; BALDISSERA, 2003). Bartolomeu Neto et al. 
(2003), investigando nadadores do sexo masculino, com suplementação de glutamina, não observaram melhora no desempenho após quatro semanas de suplementação em três esforços máximos de 100 metros nado crawl. Daniel e Cavaglieri (2005a), em um estudo com atletas jovens de futebol, não observaram melhora no desempenho físico após suplementação de 5g/dia de L-glutamina, administrados diariamente após os treinamentos. Em outro estudo com atletas de futebol, Daniel e Cavaglieri (2005b), observando o sistema imunológico, não verificaram nos suplementados com glutamina melhora na resposta imunológica, em comparação ao placebo.

A suplementação de glutamina, que é feita em praticantes de exercícios com sobrecarga e para melhora de desempenho esportivo, ainda não se apresenta com efetiva sustentação científica conclusiva (NOVELLI, et al., 2007). Assim, o presente estudo teve como objetivo investigar os efeitos da suplementação oral de glutamina sobre o desempenho de nadadores de meio-fundo e fundo.

\section{Método}

Participaram do estudo dez nadadores do sexo masculino, pertencentes a um clube do interior do estado de São Paulo, com idades entre 13 e 18 anos, todos com experiência de no mínimo dois anos na técnica de nado crawl, com no mínimo de dois anos de natação e participantes de campeonatos oficiais da Confederação Brasileira e da Federação Paulista de Natação/ Brasil, sendo todos especialistas das provas de meio-fundo e fundo ( 400 metros/800 metros). Os voluntários receberam orientação sobre a pesquisa e assinaram o Termo de Consentimento Livre e Esclarecido. O projeto de pesquisa foi aprovado pelo Comitê de Ética da Instituição onde foi realizado o estudo (protocolo $\mathrm{n}^{\mathrm{0}} 33 / 03$ ).

Para descartar contraindicações aos testes e ao treinamento físico, os nadadores foram inicialmente submetidos a uma avaliação médica, por meio de anamnese clínica e exame físico (PORTO, 2005).

Os participantes foram divididos aleatoriamente em dois grupos: grupo placebo (GP; $\mathrm{n}=5$ ), com ingestão de 5 gramas/dia de amido de milho durante 30 dias; e grupo glutamina (GG; $n=5)$, com ingestão de 5 gramas/dia de L-glutamina durante 30 dias após o treinamento. A caracterização da amostra está na Tabela 1. 
Tabela 1: Médias e desvios-padrão da idade, peso e estatura dos grupos placebo (GP) e glutamina (GG)

\begin{tabular}{lcc}
\hline & Grupo placebo $(\mathrm{n}=5)$ & Grupo glutamina $(\mathrm{n}=5)$ \\
\hline Idade & $15,6 \pm 1,5$ & $15,8 \pm 2,0$ \\
Peso & $65,8 \pm 6,8$ & $71,0 \pm 6,3$ \\
Estatura & $176,9 \pm 7,8$ & $180,1 \pm 4,1$ \\
\hline
\end{tabular}

Para a distribuição das substâncias aos respectivos grupos foi utilizada a técnica do duplo-cego, ou seja, os atletas receberam os frascos de um dos pesquisadores, o qual não teve contato com a preparação do produto. A duração da intervenção foi de 30 dias.

Os atletas foram submetidos à avaliação dietética, antes do treinamento, por meio de um formulário recordatório de sete dias, em que foram calculados a necessidade energética total (NET), segundo a Food and Agriculture Organization - World Health Organization (FAO/WHO) (VANNUCCI et al., 1990), e o valor energético total (VET). Tal recordatório permitiu que dietas individuais fossem oferecidas de acordo com as necessidades de cada atleta.

$\mathrm{Na}$ avaliação do desempenho, os nadadores foram submetidos aos testes de 800 metros e limiar anaeróbio (Lan), na técnica de nado crawl, em piscina de 25 metros, antes e após os 30 dias de intervenção. Nos testes de 800 metros considerou-se o tempo total em segundos.

A determinação do limiar anaeróbio foi por meio de testes progressivos caracterizados por dois nados no estilo crawl, em que os atletas executaram dois esforços de 400 metros: o primeiro a $90 \%$ e o segundo a $95 \%$ do seu melhor tempo na distância, com coletas de sangue efetuadas em três momentos após os testes para dosagem do lactato. A intensidade do Lan foi obtida pelo método de interpolação linear (MADER; HECK; HOLLMANN, 1976) e entre os esforços os atletas descansavam 15 minutos. As análises de lactato foram feitas retirando-se amostras de sangue do lóbulo da orelha do atleta nos seguintes tempos: um, três e cinco minutos após o esforço. Utilizou-se, para a análise da concentração de lactato, um capilar graduado para 25 microlitros e Lactímetro Accusport ${ }^{\circledR}$, sendo a velocidade do limiar determinada em metros por segundo (PELLEGRINOTTI et al.,2002).

Para avaliar a pressuposição de normalidade das variáveis tempo total no teste de 800 metros e velocidade do limiar anaeróbio, utilizouse o teste de Shapiro-Wilks. Os resultados foram descritos em média e desvio-padrão. Para comparação dos resultados antes e após a inges- 
tão de placebo ou glutamina, foi utilizado o teste t Student para dados pareados. $\mathrm{O}$ valor de $\mathrm{p} \quad 0,05$ foi considerado significante.

\section{Resultados}

As variáveis obtidas nos testes físicos apresentaram distribuição normal. Os resultados dos atletas nos testes dos 800 metros e do limiar anaeróbio apontam não haver diferenças significativas entre o primeiro e o segundo teste em ambas as variáveis (Tabela 2).

Tabela 2: Médias e desvios-padrão obtidos no primeiro e no segundo teste de 800 metros, limiar anaeróbio dos nadadores dos grupos GP e GG e resultados do teste $\mathrm{t}$ das variáveis

\begin{tabular}{lllllll}
\hline \multicolumn{1}{c}{ GP } & \multicolumn{4}{c}{ GG } \\
\hline Variável & $\mathbf{1}^{\mathbf{0}}$ teste & $\mathbf{2}^{\mathbf{0}}$ teste & $\boldsymbol{P}$ & $\mathbf{1}^{\mathbf{0}}$ teste & $\mathbf{2}^{\mathbf{0}}$ teste & $\boldsymbol{P}$ \\
TT $(\mathrm{s})$ & $661,0 \pm 32,5$ & $664,4 \pm 32,0$ & 0,776 & $616,4 \pm 16,3$ & $627,8 \pm 35,4$ & 0,255 \\
LA (m/s) & $1,18 \pm 0,10$ & $1,19 \pm 0,05$ & 0,934 & $1,27 \pm 0,05$ & $1,28 \pm 0,07$ & 0,757 \\
\hline
\end{tabular}

TT (s): tempo total no teste de 800 metros, em segundos; LA (m/s): velocidade do limiar anaeróbio em metros por segundo.

\section{Discussão}

A avaliação do desempenho dos atletas se deu na mesma fase periodizada de treinamento enquanto perdurou a pesquisa, isto é, na preparatória, com duas horas de treinos diários, de segunda-feira a sábado, totalizando 12 horas de treinos de natação por semana. Os resultados obtidos refletem o desempenho no início da preparação dos nadadores, que eram juvenis, abaixo da performance de nadadores proficientes.

A limitação do estudo foi que mesmo fazendo uma recomendação de dieta, não foi controlada a ingestão alimentar diária dos nadadores durante a suplementação de placebo e de glutamina, de modo que não se pode afirmar que os atletas seguiram as orientações nutricionais indicadas. Sabe-se, infelizmente, que o acompanhamento diário da alimentação de uma equipe é difícil de ser realizado na prática.

Após a intervenção da suplementação alimentar, os resultados dos teste físicos não apresentaram diferenças significantes entre o primeiro e o segundo teste, no tempo correspondente ao nado dos $800 \mathrm{me}-$ tros crawl, no GG e GP, indicando que a suplementação com glutamina não proporcionou melhora no desempenho físico. 
Os resultados obtidos nos testes do Lan não apresentaram diferenças significantes antes e após os 30 dias de intervenção, demonstrando que não houve melhora da performance dos nadadores com a suplementação.

Garcia, Pithon-Curi e Curi (2000) citam que parece existirem dados suficientes que provam que o exercício influencia a síntese, a liberação e a concentração plasmática de glutamina. Alterações drásticas no metabolismo muscular em consequência do exercício físico intenso e/ou prolongado podem modificar a taxa de produção/liberação de glutamina, levando a uma redução significante na concentração sérica desse aminoácido pós-atividade (CASTELL, 2003; FLAK et al., 2003), e, consequentemente, a diminuição da resistência imunológica. Uchida et al., (2006), investigando indivíduos submetidos a exercícios com pesos, observaram redução da glutaminemia somente nos testes aplicados após oito semanas de treinamento.

Entretanto, os efeitos benéficos da suplementação de glutamina na melhoria do desempenho de atletas não estão comprovados (FLAK et al., 2003). Embora seja conhecido que quando ocorre aumento da demanda de glutamina no organismo o tecido muscular eleva sua produção e liberação (PERES, 2004), tendo uma atuação importante na funcionalidade do sistema imunológico (CURY-BOAVENTURA, et al., 2008; LAGRANHA, et al., 2008), paradoxalmente esse aumento na disponibilidade parece não ser suficiente para a melhoria do desempenho físico.

A avaliação dos efeitos da suplementação oral crônica de glutamina nos nadadores de meio-fundo e fundo, em dois momentos, com testes para a capacidade aeróbia, indicou que a suplementação preconizada não proporcionou benefício aos nadadores submetidos ao teste de 800 metros. Os tempos conseguidos nos dois testes não foram significativos em comparação aos resultados do grupo controle, que foi suplementado com placebo. Todos os testes foram realizados na mesma etapa de treinamento, e esse controle evidencia que a glutamina, na dosagem administrada, não proporcionou melhora na performance dos nadadores no período preparatório.

Os resultados estão de acordo com o estudo de Bartolomeu Neto et al. (2003), realizado com nadadores do sexo masculino, após quatro semanas de suplementação de glutamina. Esse trabalho não observou melhora no desempenho dos nadadores em três esforços de 100 metros nado crawl. Os autores concluíram que a suplementação de gluta- 
mina não proporcionou benefícios no desempenho dos nadadores submetidos a teste de alta intensidade. Esses dados são corroborados pelos resultados do presente estudo em relação à suplementação de glutamina em nadadores fundistas.

Daniel e Cavaglieri (2005b) também não observaram melhora no desempenho físico de atletas jovens de futebol que foram suplementados com a mesma dose de glutamina. Outros estudos, relativos à suplementação, indicaram não haver melhoria no desempenho de atletas (FERREIRA; RIBEIRO; SOARES, 2001; MORAES et al., 2004). Porém, Oliveira et al. (2006) encontraram correlação positiva da suplementação de proteína e carboidrato na força e na massa muscular. $\mathrm{O}$ mesmo ocorreu com o estudo de Kersick et al. (2006), na avaliação de sobrecarga, com uma repetição máxima (1RM) após 10 semanas de treinamento. Cury-Boaventura et al. (2008) verificaram que maltodextrina associada com glutamina preveniu parcialmente a apoptose de linfócitos induzidos pelo exercício extenuante (maratona).

No presente estudo, a não melhora dos resultados técnicos dos nadadores de meio-fundo e fundo pode ser devida ao fato de os atletas não terem seguido a orientação dietética, uma vez que não houve um controle diário rígido da dieta deles. Assim, a glutamina talvez não tenha sido utilizada pelo organismo como suplemento alimentar, e sim como próprio alimento, complementando o valor calórico deficitário, ou mesmo atuando na sua função prioritária que é servir de nutriente para os leucócitos e os desvios para células intestinais. Assim, ao se estudarem os efeitos ergogênicos sobre a performance, com a utilização de suplementos, é fundamental verificar se eles foram utilizados como suplemento ou como complemento alimentar.

Os resultados deste trabalho corroboram o estudo de Daniel e Cavaglieri (2005a), que propõe que se a amostra pesquisada apresentar déficit do estado nutricional, isto é, em peso e composição corporal com ingesta alimentar inadequada, é possível mascarar todos os resultados em testes com o uso de suplementos ergogênicos. Assim, qualquer nutriente extra pode ser desviado metabolicamente com finalidade compensatória. $\mathrm{O}$ aspecto nutricional dos atletas deve ser controlado no dia a dia quando se pretende aplicar uma suplementação; no entanto, ressalta-se a dificuldade de realizar esse acompanhamento em uma equipe de treinamento.

Indivíduos praticantes de exercícios físicos muitas vezes podem ser estimulados, por meio de propaganda, a fazerem uso de suplementos 
nutricionais por conta própria, sem a orientação de um profissional. Assim sendo, os resultados do presente estudo devem servir de alerta para que essa prática não seja adotada.

Os nadadores desta pesquisa foram submetidos a avaliações clínicas, dietética e de esforço físico; foram orientados ao uso de dietas individuais elaboradas de acordo com as suas necessidades, embora não controladas diariamente durante a intervenção; foram submetidos a um treinamento físico supervisionado e, mesmo assim, a suplementação de glutamina não melhorou a performance, corroborando os achados de Novelli et al. (2007). Dessa forma, permanece o questionamento da efetividade da glutamina na melhoria da performance de nadadores de fundo e meio-fundo.

\title{
Conclusão
}

As avaliações deste estudo sugerem que a suplementação oral de glutamina, na dose de $5 \mathrm{~g} /$ dia durante 30 dias, administrada logo após as sessões de treinamento, não influenciou na performance de nadadores de meio-fundo e fundo.

Effect of oral glutamine supplementation on exercise performance in endurance swimmers

\begin{abstract}
The purpose of this study was to determine the influence of oral glutamine supplement on exercise performance in endurance swimmers. Ten male swimmers, 13 to 18 years of age, all specialized in $400-\mathrm{m}$ and/or $800-\mathrm{m}$ distances, participated in this investigation. The athletes were divided in two groups, placebo group with five swimmers, that ingested 5/day of corn starch, and glutamine group with five swimmers that ingested $5 /$ day of glutamine. The swimmers received the intervention for 30 days. The exercise performance measures $(800-\mathrm{m}$ time trial and anaerobic threshold, crawl technique) were obtained before and after the intervention. To compare results paired t test was used and value $p \leq 0.05$. There weren't significant differences in the results of $800-\mathrm{m}$ time trial and anaerobic threshold, in both groups. These data suggest that this oral glutamine supplementation dose didn't influence exercise performance in endurance swimmers.
\end{abstract}

Keywords: Swimming. Glutamine. Performance. 
Efecto de la suplementación con glutamina oral en el rendimiento de los nadadores de la medio fondo e fondo

\section{Resumen}

El objetivo de este estudio era observar la influencia de la suplementación con glutamina oral sobre el rendimiento físico de los nadadores de media e inferior. Hemos seleccionado a 10 atletas de sexo masculino, con edades comprendidas entre 13 y 18 años, los expertos en la natación y 400 metros de eventos / o 800. Los nadadores fueron divididos en dos grupos: placebo $(\mathrm{n}=5)$ se ingiere $5 \mathrm{~g} /$ día de almidón de maíz durante 30 días y el grupo de glutamina $(\mathrm{n}=5)$ se ingiere $5 \mathrm{~g} /$ dia glutamina durante 30 días. Pruebas (gatear y $800 \mathrm{~m}$, umbral anaeróbico, utilizando el lactato componente Lan) se produjeron antes y después de la intervención. El análisis de los resultados se realizó mediante la prueba t para datos apareados y el valor de $p \leq 0,05$. No hubo diferencias significativas en el rendimiento de los atletas durante la prueba y 800 Lan en ambos grupos. Los resultados sugieren que la suplementación con glutamina oral no influyó en el rendimiento de los nadadores de media e inferior.

Palabras clave: Natación. Glutamina. El Rendimiento.

\section{Referências}

BARTHOLOMEU NETO, J.; ROCHELLE, S. L. A.; ROCHELLE, M. C. S. A.; CAVAGLIERI, C. R.; CESAR, M. C.; PELLEGRINOTTI, I. L. Avaliação dos efeitos da glutamina na potência anaeróbia de nadadores do sexo masculino. Motriz, Rio Claro, v. 9, supl. S43, 2003.

BAXTER-JONES, A. D .G.; THOMPSON, A. M.; MALINA, R. M. Growth and maturation in elite young female athletes. Sports Medicine and Arthroscopy, v. 10, p. 42-49, 2002.

CASTELL, L. Glutamine supplementation in vitro ad in vivo, in exercice and in immunodepression. Sports Medicine, Stuttgard, v. 33, n. 5, p. 323-345, 2003.

CASTELL, L.; POORTMANS, JR.; LECLERCQ, R.; BRASSEUR, M.; DUCHATEAU, J.; NEWSHOLME, E. A. Some aspects of the acute phase response after a marathon race, and the effects of glutamine supplementation. European Journal of Applied Physiology and Occupational Physiology, Berlin, v. 75, n. 1, p. 47-53, 1997.

CURI, R. Glutamina: metabolismo e aplicações clínicas e no esporte. São Paulo: Sprint, 2000. 
CURY-BOAVENTURA, M. F.; LEVADA-PIRES, A. C.; FOLADOR, A.; ALBA-LOUREIRO, T. C.; HIRABARA, S. M.; PERES, F. P.; SILVA, P. R.; CURI, R.; PITHON-CURI, T. C. Effects of exercise on leukocyte death: prevention by hydrolyzed whey protein enriched with glutamine dipeptide. European Journal of Applied Physiology and Occupational Physiology, Berlin, v. 103, n. 3, p. 289-294, 2008.

DAMSGAARD, R.; BENCKE, J.; MATHIESEN, G.; PETERSEN, J. H.; MÜLLER, J. Body proportions, body composition and puberal development of children in comparative sports. Scandinavian Journal of Medicine \& Science in Sports, Oxford, v. 11, p. 54-60, 2001.

DANIEL, J. F.; CAVAGLIERI, C. R. Efeitos da suplementação crônica de glutamina sobre a performance de atletas de futebol categoria juvenil. Revista Brasileira de Ciência e Movimento, Brasília, v. 13, n. 4, p. 57-63, 2005a.

DANIEL, J. F.; CAVAGLIERI, C. R. Suplementação de glutamina e resistência imunológica em atletas de futebol. Saúde em Revista, Piracicaba, v. 7, n. 17, p. 21-29, 2005 b.

FERREIRA, A. M. D.; RIBEIRO, B. G.; SOARES, E. A. Consumo de carboidratos e lipídios no desempenho em exercícios de ultra-resistência. Revista Brasileira de Medicina do Esporte, São Paulo, v. 7, n. 2, p. 67-74, 2001.

FLAK, D. J.; HEELAN, K. A.; THYFAULT, A. J.; KOCH, A. J. Effects of effervescent creatine, ribose and glutamine supplementation on muscular strength, muscular endurance, and body composition. Journal of Strength and Conditioning Research, Storrs, v. 17, n. 4, p. 810-816, 2003.

FONTANA, K. E.; VALDES, H.; BALDISSERA, V. Glutamina como suplemento ergogênico. Revista Brasileira de Ciência e Movimento, Brasília, v. 11, n. 3, p. 91-96, 2003.

GARCIA, JR.; PITHON-CURI, T. C.; CURI, R. Consequências do exercício para o metabolismo de glutamina e função imune. Revista Brasileira Medicina do Esporte, São Paulo, v. 6, n. 3, p. 99-107, 2000. 
KERSICK, C. M.; RASMUSSEN, C. J.; LANCASTER, S. L.; MAGU, B.; SMITH, P.; MEITON, C.; GREENWOOD, M.; ALMADA, A. L.; EARNEST, C. P.; KREIDER, R. B. The effects of protein and aminoacid supplementation on performance and training adaptations during tem week of resistence training. Journal of Strength and Conditioning Research, Storrs, v. 20, n. 3, p. 643-653, 2006.

LAGRANHA, C. J.; LEVADA-PIRES, A. C.; SELLITTI, D. F.; PROCOPIO, J.; CURI, R.; PITHON-CURI, T. C. The effect of glutamine supplementation and physical exercise on neutrophil function. Amino Acids, Netherlands, v. 34, p. 337-346, 2008.

LEHMKUHL, M.; MALONE, M.; JUSTICE, B.; PISTILLI, E.; VINCI, D. The effects of 8 weeks of creatine monohydrate and glutamine supplementation on body composition and performance measures. Journal of Strength and Conditioning Research, Storrs, v. 17, n. 3, p. 33-38, 1992.

MADER, A.; HECK, H.; HOLLMANN, W. Evaluation of lactic acid anaerobic energy contribution by determination of post-exercise lactic concentration of ear capillary blood in middle-distance runners and swimmers. Exercise Physiology, Miami, v. 4, p.187-199, 1976.

MORAES, M. R.; SIMÕES, H. G.; CAMPBELL, C. S. G.; BALDISSERA, V. Suplementação de monoidrato de creatina: efeitos sobre a composição corporal, lactacidemia e desempenho de nadadores jovens. Motriz, Rio Claro, v. 10, n. 1, p. 17-24, 2004.

NIEMAN, D. C. Marathon training and immune function. Ports Medici, v. 37, n. 4-5, p. 412-415, 2007.

NOVELLI, M.; STRUFALDI, M. B.; ROGERO, M. M.; ROSSI, L. Suplementação de glutamina aplicada à atividade física. Revista Brasileira de Ciência e Movimento, Brasília, v. 15, n. 1, p. 109-117, 2007.

OLIVEIRA, P. V.; BATISTA, L.; MOREIRA, F.; LANCHA JR., A. H. Correlação entre a suplementação de proteína e carboidrato e variáveis antropométricas e de força em indivíduos submetidos a um programa de treinamento com pesos. Revista Brasileira de Medicina e Esporte, São Paulo, v. 12, n. 1, p. 51-55, 2006. 
PELLEGRINOTTI, I. L.; CESAR, M. C.; ROCHELLE, S. L. A.; ROCHELLE, M. C. S. A.; CAVAGLIERI, C. R. Determinação de uma intensidade de esforço para treinamento de natação de longa duração. Revista Brasileira de Ciências do Esporte, Campinas, v. 24, n. 1, p. 117-125, 2002.

PERES, F. P. Efeitos da suplementação de glutamina peptideo e carboidratos na performance de triatletas de alto nível. 2004. Dissertação (Mestrado em Performance Humana) - Universidade Metodista de Piracicaba, Piracicaba, 2004.

PORTO, C. C. Semiologia médica. 5. ed. Rio de Janeiro: Guanabara Koogan, 2005.

PREISER, J. C.; PERES-BOTA, D.; EISENDRATH, P.; VICENT, J. L.; GOSSUM, A. V. Gut mucosal and plasma concentrations of glutamine: a comparison between two enriched enteral feeding solutions in critically ill patients. Nutricion Journal, v. 2, n. 13, p. 1-5, 2003.

SILVA, C. C.; GOLDBERG, T. B. L.; TEIXEIRA, A. S.; MARQUES, I. O exercício físico potencializa ou compromete o crescimento longitudinal de crianças e adolescentes? Mito ou verdade? Revista Brasileira de Medicina do Esporte, São Paulo, v. 10, n. 6, p. 520-524, 2004.

UCHIDA, M. C.; AOKI, M. S.; NAVARRO, F.; TESSUTI, D. V.; BACURAU, R. F. P. Efeitos de diferentes protocolos de treinamento de força sobre parâmetros morfofuncionais, hormonais e imunológicos. Revista Brasileira de Medicina do Esporte, São Paulo, v. 12, n. 1, p. 21-26, 2006.

VANNUCCI, H.; MENEZES, E. W.; CAMPANA, A. O.; LAJOLO, F. $M$. Aplicações das recomendações nutricionais adaptadas à população brasileira. Ribeirão Preto: Legis Suma, 1990. p. 45.

WALSH, N. P.; BLANNIN, A. K.; CLARK, A.; ROBSON, P. J.; GLEESON, M. The effects of hight-intensity intermittent exercise on the plasma concentrations of glutamine and organic acids. European Journal Applied and Occupational Physoloy, Berlin, v. 77, n. 5, p. 434-438, 1998. 
Recebido em: 14/12/2010

Revisado em: 04/04/2011

Aprovado em: 11/08/2011

\section{Endereço para correspondência}

ilpelleg@unimep.br

Idico luiz Pellegrinotti

Universidade Metodista de Piracicaba, Mestrado Em Educação Física

Rodovia do Açúcar Km 156

Taquaral

13400-901 - Piracicaba, SP - Brasil - Caixa-Postal: 68 